\title{
Non-equilibrium systems and foundations of quantum physics
}

\author{
Václav Špička ${ }^{1, a}$, Peter D. Keefe ${ }^{2}$, and Theo M. Nieuwenhuizen ${ }^{3}$ \\ 1 Institute of Physics, Czech Academy of Sciences, Prague, Czech Republic \\ 2 University of Detroit Mercy, Detroit, USA \\ 3 Institute for Theoretical Physics, University of Amsterdam, Amsterdam, The Netherlands
}

Received 8 March 2021 / Accepted 8 March 2021 / Published online 27 May 2021 (C) EDP Sciences, Springer-Verlag GmbH Germany, part of Springer Nature 2021

\begin{abstract}
Presented is a brief overview of recent developments of topics addressed by the reviews and original papers of this volume related to non-equilibrium phenomena in various (especially mesoscopic) systems and the foundations of quantum physics.
\end{abstract}

\section{Introduction}

The volume summarizes advances in the understanding of the behavior of systems out of equilibrium, together with related themes of the foundation of quantum physics and quantum thermodynamics. The original contributions are from top scientists of these fields (and their collaborators) who participated in the conference Frontiers of Quantum and Mesoscopic Thermodynamics (FQMT'19), which was organized by the Institute of Physics of the Czech Academy of Sciences and was held in Prague in July of 2019, and was a follow-up to the six previous FQMT conferences (https://fqmt.fzu. $\mathrm{cz} /$ ), see also [1-6].

The volume contains review papers and original papers which provide readers an excellent orientation to the state-of-the-art of the following topics:

$\diamond$ Non-equilibrium statistical physics and quantum transport;

$\diamond$ Quantum thermodynamics and engines;

$\diamond$ Foundations of quantum physics;

$\diamond$ Quantum optics, light-matter interactions;

$\diamond$ Quantum many body systems, phase transitions, superfluidity, superconductivity;

$\diamond$ Quantum computing;

$\diamond$ Biophysics and chemical physics; and

$\diamond$ Gravitation and cosmology.

We believe that this set of papers provides useful insights into recent developments in the abovementioned topics and at the same time, the volume documents important relations between them.

Recent advances in technologies, which have led to enormous improvements of measurement, imaging and observation techniques at microscopic, mesoscopic and

\footnotetext{
a e-mail: spicka@fzu.cz (corresponding author)
}

macroscopic scales, have accelerated the development of these topics and their ever-increasing mutual influence as to the use of both theoretical and experimental techniques, as well as understanding of the quickly increasing number of observed phenomena.

Moreover, nowadays, experimental as well as theoretical investigations of time evolution of classical and quantum systems far from equilibrium are quickly developing. Various methods now allow investigation into time evolution of non-equilibrium systems at different time scales. More detailed observations of non-equilibrium systems behavior bring, however, also increasing pressure on attaining a better understanding and a more detailed description of equilibrium features of investigated systems since their knowledge is requisite as a point of entry into deeper studies of non-equilibrium behavior. Therefore, this volume contains, apart from papers which deal with time evolution, papers devoted to systems in equilibrium.

From the point of view of experimental and observational possibilities, the understanding of few body systems, as well as many body systems, both in and out of equilibrium, is far from complete. The increasing ability to study subtle details of equilibrium features and, at the same time, the dynamics of systems, creates new challenges in many fields of physics. To cope successfully with these challenges means seeking a more detailed understanding of phenomena, interactions and parameters which play key roles in determining system behavior, such as proper determination of interaction strengths, influence of size and geometry of systems, quantum correlations, entanglement and their attendant dynamics; decoherence and dissipation; light-matter interactions; equilibration and thermalization of systems; roles of initial and boundary conditions; influence of the environment, strength of systemenvironment interactions, reservoirs and external fields on the time evolution of systems; quantum to classi- 
cal transitions; dynamics of quantum phase transitions; and topological states of systems. The above-mentioned challenges occur not only in many fields of physics, but also in astrophysics, chemistry, and biology. Thus, this volume includes several papers which are at the border of physics and other natural sciences.

Mesoscopic systems are of special importance for these studies. Various systems, of both natural and artificial origin, can exhibit mesoscopic features depending on inherent inner parameters and interactions with their environment. Typical mesoscopic systems are of nanometer size. Fast-developing nanoscale technologies enable us to prepare an enormously diverse range of systems with well defined inner parameters and with good control of their interactions with the external environment and time-dependent fields.

Nanoscale structures include not only very small artificially prepared structures, but also structures occurring in living cells, as for example complex molecules, proteins and molecular motors. Such systems are on the borderline between different disciplines (i.e., physics, chemistry, and biology) where the dynamic behavior of these systems and corresponding various methods of their description (individual or statistical, microscopic or macroscopic, classical or quantum) meet. These (often open) systems are commonly dominated by quantum effects, by topology of their structures and states, and by strong interactions with their environment. Due to their position between the macro and micro world, these systems exhibit many surprising phenomena which can lead to a better understanding of quantum mechanics, many-body physics, quantum computing, and the relation between classical and quantum behaviors by sensitive choice of parameters. The development of theoretical concepts for their description and reliable experimental methods is of great importance for investigating these systems, testing theories and designing new nanostructures with well-defined, desired behavior. They can be studied by methods of condensed matter physics and quantum optics in such detail that affords a deeper understanding of quantum physics, as represented by quantum interferences, entanglement, the uncertainty principle and quantum measurement processes. The specific arrangements and the control of the number of constituent particles in systems featuring cold atoms and molecules can reveal macroscopic quantum effects and can be used for testing foundations of quantum physics and methods of quantum many-body theory.

Studies of these systems can shed new light on longstanding and extremely important problems for better understanding of observed phenomena in many fields of the natural sciences, such as dynamics of measurement processes; relations between systems parameters, system-environment interactions, external fields and timescales of system evolution; irreversible behavior of real systems in comparison with reversible microscopic laws; emergence of classical macroscopic behavior from microscopic quantum behavior; limits to "phenomenological" thermodynamic descriptions, the problem of how to describe properly open quantum systems far from equilibrium, especially in the case of strong interaction between a small system and reservoirs; stochastic behavior of systems, which can be caused either by innate features of the studied systems or by the fact that the studied system is open.

Studies of various system parameters and their influence on the behavior of systems, together with investigations of related quantum and temperature fluctuations, quantum noise, dephasing and dissipation, create an essential part of the research which aims to better understand the performance and reliability of artificially created structures being studied, e.g., in the quickly developing field of quantum computing or of small (nanoscale, classical or quantum) engines.

Small "engines" are also represented by molecular motors in cells of living organisms, which represent key elements of cells performance. Their discovery opened many, up to now not yet satisfactorily answered, questions on the way to understanding the laws underlying life. It is now evident that the performance of living cells is based on (in general) far from equilibrium states and that non-equilibrium processes play a decisive role in the proper performance of various structures which are essential for life. One of the key features of complex organisms is their ability to reach homeostasis. Accordingly, a proper understanding of relaxation, equilibration, steady state and local (in time and space) equilibrium states of various complex systems in cells and their interactions with the environment is critical. Thus, non-equilibrium statistical physics and thermodynamics can help on the way to understanding life processes. Another essential aspect of living organisms involves networks (as for example neural or immune systems), which depend on synchronization of the inner structures of various cells and their mutual interactions. Therefore, the volume contains manuscripts which deal with these aspects of life from the point of view of the involved physical mechanisms.

In general, the above-mentioned problems arise in dissipation, dephasing and decoherence processes, and, on a very basic level, the foundations of quantum mechanics and measurement.

Historically, classical thermodynamics employed phenomenological treatments to introduce the concepts of "temperature", "system", "reservoir", and "engine". The central question nowadays is under what conditions will thermodynamic behavior still manifest in various small systems. Due to the quantum aspects of small (molecular) systems, it is necessary to deal with quantum thermodynamics to properly discuss quantum pumps, quantum heat engines and quantum refrigerators. The task of quantum thermodynamics is to provide a suitable "phenomenological" framework, such as the "ergotropy", for the "macroscopic" description of open mesoscopic systems, coming from more detailed studies of non-equilibrium quantum statistical physics of open systems and the foundations of quantum mechanics.

A better knowledge and insight into the foundations of quantum and non-equilibrium statistical physics is essential for a proper formulation of the fundamen- 
tal laws of physics, astrophysics and cosmology with regard to Bell inequalities and quantum gravity. It is also essential for developing a suitable description of small quantum systems and their applications. This applies particularly to quantum optics and the physics of quantum information and computing, where detailed understanding of quantum interference, entanglement and decoherence processes, together with related knowledge of timescales governing the dynamics of the studied systems, are essential.

The contributions to this topical issue have been grouped in eight sections, as indicated above, and in the contents of the volume. Details of recent developments regarding the subjects of the individual sections can be found in the included reviews and papers, and references therewith. Additional information can be also found in journal volumes dedicated to the previous FQMT conferences [1-6]. We trust that in this way the reader has been provided a working orientation to the topics contained in this volume.

Acknowledgements We are indebted to Peter Hänggi, Sabine Lehr, and Sandrine Karpe for all their support, efforts and professional work throughout the preparation of this special issue. We would also like to thank all other members of EPJ ST for their help.

\section{References}

1. Th.M. Nieuwenhuizen, P.D. Keefe, V. Špička (eds.), Proceedings of the International Conference on Frontiers of Quantum and Mesoscopic Thermodynamics (FQMT'04), Physica E 29, N. 1-2, (2005)

2. Th.M. Nieuwenhuizen, P.D. Keefe, V. Špička (eds.), Proceedings of the International Conference on Frontiers of Quantum and Mesoscopic Thermodynamics (FQMT'08), Physica E 42, N. 3, (2010)

3. Th.M. Nieuwenhuizen, P.D. Keefe, V. Špička (eds.), FQMT'11: Topical Issue-International Conference on Frontiers of Quantum and Mesoscopic Thermodynamics, Physica Scripta T151 (2012)

4. Th.M. Nieuwenhuizen, P.D. Keefe, V. Špička (eds.), FQMT'13: Topical Issue-International Conference on Frontiers of Quantum and Mesoscopic Thermodynamics, Physica Scripta T165 (2015)

5. P.D. Keefe, Th.M. Nieuwenhuizen, V. Špička (eds.), Topical Issue-International Conference on Frontiers of Quantum and Mesoscopic Thermodynamics (FQMT'15), Fortschritte der Physik (Progress of Physics) 65, N. 6-8 (2017)

6. P.D. Keefe, Th.M. Nieuwenhuizen, V. Špička (eds.), Nonequilibrium dynamics: quantum systems and foundations of quantum mechanics. Eur. Phys. J. Spec. Top. 227, N. 15-16 (2019) 